\title{
Medición de la eficiencia técnica relativa de las fincas asociadas a Coounión en Guasca Cundinamarca
}

\section{Measurement of the relative technical efficiency of the farms associated to Coounion in Guasca Cundinamarca}

\author{
Wilson Oviedo G, ${ }^{1}$ M.Sc, Gloria Rodríguez L, ${ }^{2 *}$ Ph.D.

\begin{abstract}
${ }^{1}$ Universidad Nacional de Colombia, Maestría en Administración, Ciudad Universitaria de Bogotá, Colombia. ${ }^{2}$ Universidad Nacional de Colombia, Facultad de Ciencias Económicas, Ciudad Universitaria
\end{abstract} \\ de Bogotá, Colombia. *Correspondencia: girodriguezl@unal.edu.co.
}

Recibido: Mayo de 2010; Aceptado: Febrero de 2011.

\section{RESUMEN}

Objetivo. Medir la eficiencia técnica relativa en las fincas asociadas a la cooperativa COOUNIÓN mediante Data Envelopment Analysis DEA (Análisis Envolvente de Datos). Materiales y métodos. Se tomaron como muestra las doce fincas asociadas a la Cooperativa Coounión en el municipio de Guasca (Cundinamarca). La información se obtuvo durante el período comprendido entre el $1^{0}$ de junio del 2008 y el 31 de mayo del 2009. Los datos se estructuraron en seis variables, de las cuales se plantean dos modelos, ambos con cuatro variables y que están orientados a las entradas con rendimientos constantes a escala (CRS). El primer modelo consta de tres entradas: nutrición, mantenimiento, ordeño, y una salida: leche; mientras que el segundo modelo cuenta con dos entradas: nutrición, mantenimiento y dos salidas: carne, crías. Resultados. De las doce fincas solo una presentó, la mejor eficiencia tanto en el modelo 1 como en el modelo 2. Por esta razón, se determinaron las mejores prácticas de esta finca con el fin de replicarlas en las demás. Conclusiones. Aplicar la metodología DEA en las fincas ganaderas es viable porque permite enfocarse principalmente en aquellas variables que son controlables por el ganadero como los insumos. Por consiguiente, la evaluación de la eficiencia técnica relativa se desarrolló orientada a estos; así se demostró que las fincas pueden mantener sus niveles de producción actuales haciendo reducciones significativas en sus costos.

Palabras clave: Bovinos, carne de res, mejoramiento animal, nutrición animal, ordeño. (Fuente: AIMS). 


\section{ABSTRACT}

Objective. To determine the relative technical efficiency using the data envelopment analysis DEA of farms associated to the Coounion. Materials and methods. A total of 12 farms associated to the Coounion Cooperative, municipality of Guasca (Cundinamarca) were studied. Data were obtained during a period of one year (June/01/2008 to May/31/ 2009) and structured in six variables, of which two models both appear with four variables that are oriented to the inputs with constant scale returns (CRS). The First Model consists of three entries: Nutrition, Maintenance, Milking, and one output: Milk. the Second Model has two entrances: Nutrition, Maintenance, and two outputs: Meat and Mreeding. Results. From the twelve farms studied only one presented the best efficiency, for both models (Model 1 and 2). Therefore, the best practices of this farm were considered to be applied on the others farms Conclusions. To apply the methodology DEA in cattle farms is viable because it allows to focus mainly in those variables that are controllable by the rancher like the consumables. Therefore, the evaluation of the relative technical efficiency was developed oriented to the consumables. It was demonstrated that the farms can maintain their present levels of production making significant reductions in their costs.

Key words: Animal breeding, animal nutrition, beef, bovine, milking. (Source: AIMS).

\section{INTRODUCCIÓN}

El ganado bovino desciende de especies salvajes que existieron en Europa y Asia en tiempos muy remotos. Con el tiempo, el hombre después de un periodo largo de domesticación, logró su dominio vinculándolo a su economía dada la variedad de productos que de éste podía obtener (1).

Asimismo, la ganadería en Colombia es una actividad productiva desde los tiempos de la conquista a mediados del siglo XVI, cuando los españoles introdujeron las primeras cabezas de ganado al país. De hecho, ellos traían sus ganados, tomaban y elegían las mejores tierras para poner sus granjerías y aprovechaban las labores de las mujeres indígenas para la crianza de estos animales (2).

Precisamente, la ganadería en los años de la conquista existía en el norte del continente americano y en el área de influencia del Imperio Inca con especies como el bisonte de Norteamérica y los camélidos medianos respectivamente. No obstante, el ganado bovino entró a Colombia por la Costa Atlántica provenientes de las islas del Caribe, entre estas la isla Española y la isla Margarita. Desde luego, el ganado existente en estas islas fue puesto allí por Cristóbal Colón y sus acompañantes (3).
Entonces la historia del ganado bovino en el Nuevo Mundo es reciente, porque no existía en el momento del descubrimiento por los primeros colonos españoles. De ahí que, los españoles introdujeron al país las razas: Andaluza, Pirenaica, Tundaca, Gallega, Berrenda Andaluza, Cacereña y Murciana. Por este motivo, las poblaciones bovinas en Colombia se constituyeron progresivamente a partir de estas importaciones diversas según la historia propia de cada región. Es así, como se desarrollaron y formaron las razas criollas en el país; a partir de la evolución de las razas traídas por los conquistadores (4).

Sin embargo, la ganadería adquiere importancia económica desde la primera mitad del siglo XX. A partir de esa época, en Colombia se importaron razas europeas como: Holstein, Normando, Pardo Suizo, Ayrshire, Jersey, Limousin y Simmental; además de la raza Cebú originaria de TexasEstados Unidos, que se empleó a mediados del siglo pasado para cruzarla con razas europeas; y hacerlas más resistentes al clima tropical (3).

Pero, esta actividad productiva ha sido muy golpeada por el conflicto armado. Por tanto, las condiciones de inseguridad extrema 
en las áreas rurales, dan lugar a procesos significativos de expulsión de población campesina de sus parcelas, convirtiéndolos en grupos itinerantes de desplazados (5).

Igualmente, el calentamiento global es un flagelo que no solo afecta a la ganadería sino a la economía en general. Ya que, este es el fenómeno natural que por degradación del medio ambiente ha llevado a lluvias extremas y oleadas de calor sin precedentes (6). Esto significa, que hay lugares en la tierra que se van a calentar, mientras que otros se van a enfriar (7).

Indistintamente, la ganadería se afecta por el cambio climático porque se eleva la temperatura global; calentando los océanos, dando lugar a evaporación de los mares que genera nubes que sustraen la humedad al suelo haciéndolos desérticos e improductivos para el alimento de estos animales. Por ende, los lugares que fueron escogidos para la explotación de ganado bovino por su raza, están cambiando y haciéndose cada vez menos aptos para la cría de este ganado (6).

En síntesis, estos cambios atmosféricos conllevan a la escasez de forrajes, agua y suplementos alimenticios. En consecuencia, "cuando la alimentación es deficiente en cantidad y calidad, el estado de salud del ganado se ve perjudicado. Surgen entonces alteraciones de todo tipo, tales como: disminución de la eficiencia productiva, retardo del crecimiento corporal, baja producción de leche, falta de vivacidad, pelo sin brillo y erizado, pérdida de peso, baja en las defensas orgánicas favoreciendo la aparición de enfermedades infecciosas" (8).

Por las anteriores razones, es necesario hacer más eficientes los procesos productivos en las fincas ganaderas. Porque la ganadería aporta al crecimiento económico del país; entonces, es viable aprovechar los avances científicos en las ciencias administrativas y económicas a favor de la industria bovina.

Teniendo en cuenta que Data Envelopment Analysis DEA (Análisis Envolvente de Datos) es la técnica de programación lineal que examina las relaciones entre los inputs (entradas) y los outputs (salidas) utilizados en un proceso de producción para determinar los niveles de eficiencia (9), se ha determinado utilizarla en esta investigación para analizar la eficiencia técnica relativa de las fincas ganaderas en estudio.

Modelo: Data Envelopment Analysis (DEA). DEA es una técnica que facilita la medición y comparación de la eficiencia de unidades como empresas agropecuarias, universidades, escuelas, hospitales entre otras, donde haya un conjunto relativamente homogéneo de unidades. Dentro del vocabulario del DEA, estas unidades organizacionales se denominan Decision Making Units ( DMU) (10).

La medición de la eficiencia es importante para las empresas que quieren mejorar la productividad por medio de la racionalización de los recursos. Por consiguiente, las organizaciones investigan como hacer un uso adecuado de las entradas buscando encontrar el ahorro posible en la utilización de estas (11).

Posteriormente se desarrolla el modelo de Charnes, Cooper and Rhodes (CCR) que es conocido como el modelo de rendimientos constantes a escala (CRS); y el modelo de Banker, Cooper and Rhodes (BCC) que incluye rendimientos variables a escala (VRS) (12).

Los dos modelos de eficiencia se construyen por proyección radial. Concretamente, en el modelo orientado a los insumos, estos son proporcionalmente disminuidos, mientras que los productos permanecen fijos. Para el modelo orientado a las salidas, estas son proporcionalmente aumentadas, mientras que los insumos se mantienen constantes (12).

La escogencia de la orientación del problema dependerá de la naturaleza de los objetivos que se planteen. Generalmente, los usos de DEA en investigaciones de ganadería han empleado el enfoque orientado a los insumos. Este enfoque permite el diagnóstico sobre la subutilización de los recursos con la tecnología y los recursos existentes. Esto no significa que no se hayan realizado investigaciones en las que la referencia de comparación son fincas con nuevas técnicas productivas (13). 
De hecho, el modelo DEA es una alternativa para medir la eficiencia técnica, que refleja la habilidad de una DMU para obtener el máximo nivel de producción con unos recursos dados. Esta eficiencia técnica es calculada para varias DMU que emplean los mismos insumos para producir los mismos productos. Por lo tanto, DEA toma los resultados de estas eficiencias técnicas y construye una frontera eficiente que es el conjunto de soluciones que dominan estratégicamente a las demás soluciones posibles, con el fin de determinar por medio de comparaciones la eficiencia relativa de cada DMU (14).

Más aún, DEA es un programa lineal que deduce la función de frontera de producción de un conjunto de DMU, identificando la eficiencia técnica relativa de cada una de estas DMU, discriminando las eficientes de las ineficientes. Las DMU ubicadas sobre la frontera tienen una puntuación de 1 , y la ineficiencia de las demás es hallada sobre la base de la distancia Euclidiana de sus proporciones de entrada y salida de la frontera de producción (15).

Resumiendo, DEA es un modelo de optimización no paramétrico que evalúa el desempeño de las DMU. En su forma operativa básica, DEA es una metodología utilizada para medir la eficiencia comparativa de DMU que tienen un mismo fin en el mercado. Partiendo de insumos y productos, DEA provee un ordenamiento de estas DMU otorgándoles un puntaje de eficiencia relativa. De esta forma, las que obtengan el mayor nivel de producto con la menor cantidad de insumos, serán las más eficientes del grupo $y$, por ende, obtendrán los puntajes más altos (16).

Las Variables de holgura. En DEA las variables de holgura tienen una relación directa con la solución del problema. Por este motivo, si la solución óptima del problema dado por el modelo resulta ser de eficiencia 1, significa que la finca evaluada es eficiente, en relación con las otras fincas; puesto que no es posible encontrar ninguna finca que obtenga al menos las salidas de la finca evaluada utilizando menos recursos. Siendo así, la finca es ineficiente cuando su eficiencia es menor de 1; entonces, el valor de holgura resulta al restarle a 1 el valor de la eficiencia relativa de la finca ineficiente (17).

En consecuencia, toda finca de eficiencia 1 tiene un valor de holgura igual a cero. Es decir, toda finca que tenga un valor de holgura significa que tiene ineficiencias a escala. Entonces, entre mayor sea el valor de la holgura, menor será la eficiencia de la finca evaluada.

Se analizaron doce fincas asociadas a la Cooperativa Coounión en el municipio de Guasca Cundinamarca. Estas fincas, se dedican a la producción de leche; crías; y venta de ganado en pie. El objetivo del estudio fue medir la eficiencia técnica relativa de estas fincas en la producción de carne, leche y crías, en bovinos; mediante el DEA.

\section{MATERIALES Y MÉTODOS}

Tipo de estudio. El estudio de caso se realizó en las doce fincas asociadas a Coounión que se encuentran localizadas en el municipio de Guasca-Cundinamarca, Colombia. Las coordenadas geográficas donde se encuentran ubicadas estas fincas éstan entre $5^{\circ} 00^{\prime}$ de latitud Norte y $74^{\circ} 00^{\prime}$ de longitud Oeste, con una altitud de 2.800 metros sobre el nivel del mar.

Condiciones geoclimáticas. En el área geográfica donde se realizó el estudio el clima se caracteriza por una precipitación anual acumulada de 1000 $\mathrm{mm}$, temperatura media promedio anual de $14^{\circ} \mathrm{C}$, brillo solar anual acumulado de 1410 horas (con un máximo de 1700 horas y un mínimo de 1120 horas) y humedad relativa alrededor de $80 \%$ (18).

Animales de estudio. Las razas de bovinos sobre los cuales se hicieron las mediciones de consumo, al igual 
que los productos obtenidos de estos fueron: Holstein, Jersey, Pardo Suizo y Simmental.

Periodo de evaluación. Del 1 de junio de 2008 hasta el 31 de mayo de 2009.

Recolección de la información. La información se recolectó por medio de una encuesta. Se indagó los costos por finca con relación a la nutrición en lo que concierne al cuidado de los forrajes y los suplementos alimenticios como sales, concentrados, silos, henos, y agua. En cuanto a los costos de mantenimiento, se investigó sobre la sanidad entendida esta como la vacunación, la desparasitación, medicamentos, servicios veterinarios, reproducción; como también en estos costos se consideró el levante y el mantenimiento de las vacas secas. Por último, se consideraron los costos de ordeño. Toda esta información fue recogida directamente en cada finca. La información de producción de carne, leche y crías; fueron suministradas por la Cooperativa.

Mediciones. De acuerdo con los insumos analizados y los productos obtenidos de cada finca, se determinaron seis variables; entre las que se tienen como variables de entrada (insumos): nutrición, mantenimiento y ordeño. De igual manera, se determinaron las variables de salidas (productos): carne, leche y crías. Estas variables fueron consideradas partiendo de las investigaciones sobre metodologías de costos de pequeñas fincas ganaderas de la sabana de Bogotá realizadas por la corporación de estudios ganaderos y agrícolas (19). Por esta razón, no se consideraron variables como la de investigación y desarrollo y la variable de tecnología; que son más comunes en medianas y grandes explotaciones ganaderas, debido a que estas explotaciones ganaderas en su mayoría no son proveedores de leche a pasteurizadoras; sino que llevan a cabo un proceso productivo de mayor complejidad.
Las variables de entradas y salidas se cuantificaron en pesos colombianos invertidos y percibidos respectivamente, generados mes a mes. Los valores agregados por variable se dividieron por el número de vacas de ordeño del respectivo mes. Aquí es importante anotar que el peso de las salidas en orden descendente tienen las siguientes ponderaciones en la cooperativa: leche $90.19 \%$, carne $8.92 \%$ y crías $0.89 \%$.

\section{Diseño experimental y software} empleado. Con las anteriores variables, se plantearon dos modelos: el modelo 1 , que combinó entradas como nutrición, mantenimiento y ordeño con la producción de leche; y el modelo 2 combinó las entradas de nutrición y mantenimiento con la producción de carne y crías. Por lo tanto, el modelo 1 mide la eficiencia en la producción de leche y el modelo 2 calcula la eficiencia en cuanto a la producción de carne y crías.

El software empleado para correr los dos modelos fue el DEA FRONTIER, desarrollado por el profesor Joe Zhu; quien ha publicado y coeditado varios libros enfocados a la evaluación de desempeño de DMU e identificación de mejores prácticas usando el DEA. Por lo tanto, él es un experto en métodos de medición de desempeño y sus investigaciones propenden por las áreas de operaciones y productividad, evaluación de desempeño de DMU y mejores prácticas de éstas DMU. En efecto, el profesor Zhu se encuentra en el ranking de los 10 autores con más investigaciones en DEA (20).

Planteamiento matemático. Como complemento a la metodología se específica en el planteamiento matemático los problemas de programación lineal pertinentes. En consecuencia, se deben minimizar los niveles de las variables de entrada (nutrición, mantenimiento y ordeño), manteniendo el nivel en las variables de salida (leche, carne y crías).

Para el modelo 1 , de tres entradas (nutrición, mantenimiento y ordeño) y una salida (leche); el planteamiento es, 
sujeto a;

$$
\begin{aligned}
\operatorname{Min}_{\theta_{k} \lambda} \theta_{k} \quad & x_{1 k} \theta_{k}-\sum_{j=1}^{12} x_{1 j} \lambda \geq 0 \\
& x_{2 k} \theta_{k}-\sum_{j=1}^{12} x_{2 i} \lambda \geq 0 \\
& x_{3 k} \theta_{k}-\sum_{j=1}^{12} x_{3 j} \lambda \geq 0 \\
\lambda \geq \varepsilon \quad & -y_{1 k}+\sum_{j=1}^{12} y_{1 j} \lambda \geq 0
\end{aligned}
$$

El modelo 2, de dos entradas (nutrición y mantenimiento) y de dos salidas (carne y crías), se plantea, sujeto a:

Donde:

$$
\begin{array}{r}
\operatorname{Min}_{\theta_{k} \lambda} \theta_{k} \quad x_{1 k} \theta_{k}-\sum_{j=1}^{12} x_{1 j} \lambda \geq 0 \\
x_{2 k} \theta_{k}-\sum_{j=1}^{12} x_{2 j} \lambda \geq 0 \\
-y_{2 k}+\sum_{j=1}^{12} y_{2 j} \lambda \geq 0 \\
\lambda \geq \varepsilon \quad-y_{3 k}+\sum_{j=1}^{12} y_{3 j} \lambda \geq 0
\end{array}
$$

y es la variable de salida, que son los productos de cada DMU; estas son:

y1=leche, y2=carne, y3=crías.

$x$ es la variable de entrada, que son los insumos empleados por las DMU; estos son:

x1 = nutrición, $x 2$ = mantenimiento, $x 3=$ ordeño.

$\mathrm{k}$ representa una finca de las doce asociadas a COOUNIÓN.

$\theta_{k}$ escalar que multiplica el vector de las entradas (nutrición, mantenimiento y ordeño) de la finca k que se va a evaluar.

El valor minimizado de $\theta_{k}$ teniendo en cuenta las tres primeras restricciones dará como resultado la eficiencia para esta finca. $\lambda$ es el escalar que multiplica a la matriz de entradas y salidas de todas las fincas j. Su restricción vienen dada por la última ecuación que supone que esté restringida a ser mayor o igual a una cantidad mínima $\varepsilon$, con el objeto de evitar que algunas de las entradas o la propia salida o salidas (modelo 2) sean totalmente ignoradas en la determinación de la eficiencia.

$\varepsilon$ es un valor no Arquímedeo diseñado para hacer cumplir estrictamente la positividad de las variables.

\section{RESULTADOS}

Análisis de eficiencia. Las fincas son más eficientes en el modelo 1 (producción de leche); que el en modelo 2 (producción de carne y crías) (Tabla 1 ).

Tabla 1. Resultados de eficiencia modelos 1 y 2.

\begin{tabular}{cccccc}
\hline & & \multicolumn{2}{c}{ Modelo 1 } & \multicolumn{2}{c}{ Modelo 2 } \\
\hline DMU 1 & Santa Teresita & 0.68 & $2,8,11$ & 0.2 & 6,11 \\
DMU 2 & El Recuerdo & 1 & 2 & 0.37 & 11 \\
DMU 3 & Amazonas & 0.72 & $8,10,11$ & 0.49 & 6 \\
DMU 4 & El Rubí & 0.77 & $2,8,11$ & 0.63 & 6,11 \\
DMU 5 & Casa Teja & 0.75 & $8,10,11$ & 0.36 & 6,11 \\
DMU 6 & Los Pinos & 0.96 & $2,8,11$ & 1 & 6 \\
DMU 7 & Los Arrayanes & 1 & 7 & 0.53 & 6,11 \\
DMU 8 & El Porvenir & 1 & 8 & 0.79 & 6,11 \\
DMU 9 & El Desvare & 0.71 & 2,11 & 0.21 & 6,11 \\
DMU 10 & Buena Vista & 1 & 10 & 0.47 & 6,11 \\
DMU 11 & El Molino & 1 & 11 & 1 & 11 \\
DMU 12 & El Dorado & 1 & 12 & 0.67 & 6 \\
Eficiencia Media \% & 0.89 & & 0.56 & \\
\hline
\end{tabular}

Al mismo tiempo, para los dos modelos las DMU de eficiencia 1 son referenciadas a las DMU con ineficiencias; para que estas DMU mal evaluadas copien las mejores prácticas de las DMU eficientes, y así mejoren sus niveles relativos de eficiencia (Tabla 1 ).

El modelo 1, muestra que el $50 \%$ de las fincas evaluadas fueron eficientes en los rendimientos constantes a escala; significa esto, que el 50\% restante tienen ineficiencias a escala. En el modelo 2, el $17 \%$ de las fincas fueron eficientes; entonces, el 
$83 \%$ restante tienen ineficiencias a escala (Tabla 1).

La distribución de los resultados de eficiencia de los modelos 1 y 2 muestra que las eficiencias del modelo 1 se encuentran entre el rango del $61 \%$ y el $100 \%$. Sin embargo, las eficiencias del modelo 2 están en el rango del $11 \%$ al $100 \%$. De hecho, el $100 \%$ de las fincas en el modelo 1 , tienen su eficiencia por encima del $67 \%$. Situación que no se puede observar en el modelo 2, donde el $50 \%$ de las fincas tienen su eficiencia por debajo del $50 \%$ (Figura 1).

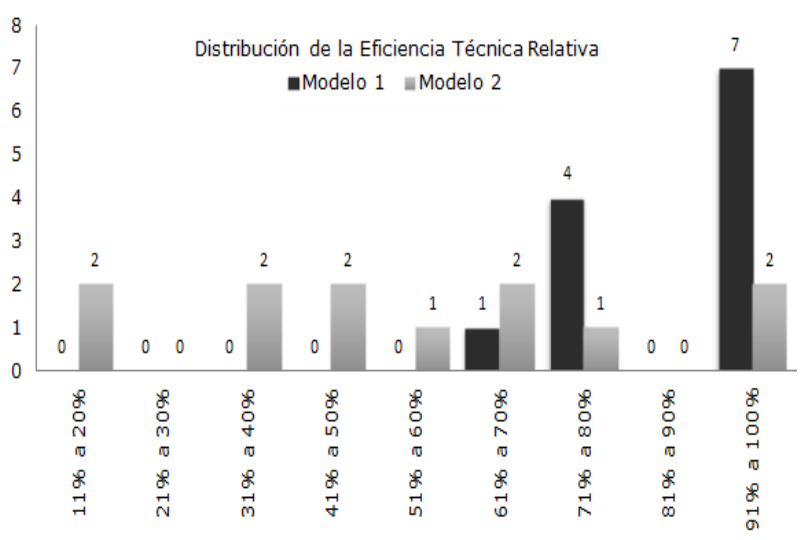

Figura 1. Distribución de los resultados de eficiencia en modelos 1 y 2.

Mejores prácticas en las DMU con eficiencia 1. Las fincas con eficiencia 1 en el modelo 1 son: EL Recuerdo, Los Arrayanes, El Porvenir, Buena Vista, El Molino y El Dorado; que corresponden a las DMU 2, 7,
$8,10,11$ y 12 respectivamente. En este modelo, el promedio de leche producida por vaca de ordeño es la característica común de estas DMU; este promedio está en el rango de 409 a 489 L/Vaca ordeñada al mes. No obstante, las fincas ineficientes que son las DMU 1, 3, 4, 5, 6 y 9; tienen el promedio en el rango de 304 a 384 L/Vaca ordeñada al mes (Tabla 2).

En tanto, el modelo 2 tiene las fincas Los Pinos y El Molino con eficiencia 1, que son las DMU 6 y 11 respectivamente. Al observar los ítems de Carne y Crías, la DMU 6 es la mayor generadora de ingresos por venta de carne en pie con $\$ 11.000 .000$ al año, aventajando en un $46 \%$ a la que más se le aproxima que es la DMU 4 Finca El Rubí que tiene ventas anuales de Carne por \$6.000.000; las demás DMU tienen ventas de Carne al año por debajo de los $\$ 3.600 .000$ (Tabla 2).

Por su parte, la DMU 11 Finca El Molino, en Crías cuenta con el valor promedio de ventas de terneros más alto, al registrar $\$ 90.000$ por ternero de Cría vendido al año; aventajando en un $20 \%$ a la DMU 4 que tiene un promedio de $\$ 72.000$ por ternero de Cría vendido al año; las demás DMU son superadas por la DMU 11 en más del 30\% en el valor de ternero de Cría vendido al año (Tabla 2).

La DMU 6 Finca Los Pinos tiene $0.96 \%$ de eficiencia en el primer modelo y eficiencia

Tabla 2. Producción de carne, leche y crías.

\begin{tabular}{cccccccc}
\hline & & \multicolumn{3}{c}{ Modelo 1 } & \multicolumn{3}{c}{ Modelo 2 } \\
\hline DMU & FINCAS & VACAS & $\begin{array}{c}\text { LECHE L/ } \\
\text { Vaca }\end{array}$ & \%EFIC & $\begin{array}{c}\text { CARNE al } \\
\text { Año (\$) }\end{array}$ & $\begin{array}{c}\text { CRÍAS (\$) } \\
\text { Prom. Año }\end{array}$ & \%EFIC \\
\hline 1 & Santa Teresita & 5 & 365 & 0.68 & 0 & 50.000 & 0.19 \\
2 & El Recuerdo & 15 & 443 & 1 & 1.600 .000 & 50.000 & 0.37 \\
3 & Amazonas & 8 & 356 & 0.72 & 3.600 .000 & 40.000 & 0.49 \\
4 & El Rubí & 12 & 371 & 0.77 & 6.000 .000 & 72.000 & 0.63 \\
5 & Casa Teja & 16 & 304 & 0.75 & 3.600 .000 & 50.000 & 0.36 \\
6 & Los Pinos & 12 & 384 & 0.96 & 11.000 .000 & 61.667 & 1 \\
7 & Los Arrayanes & 10 & 449 & 1 & 3.600 .000 & 52.000 & 0.53 \\
8 & El Porvenir & 9 & 429 & 1 & 2.800 .000 & 65.714 & 0.79 \\
9 & El Desvare & 4 & 368 & 0.71 & 1.000 .000 & 45.000 & 0.20 \\
10 & Buena Vista & 9 & 409 & 1 & 2.700 .000 & 48.000 & 0.47 \\
11 & El Molino & 4 & 455 & 1 & 2.400 .000 & 90.000 & 1 \\
12 & El Dorado & 6 & 489 & 1 & 2.800 .000 & 50.000 & 0.67 \\
\hline
\end{tabular}


1 en segundo modelo; ocupando el primer puesto en producción de carne y el cuarto en producción de crías (Tabla 2 ).

Análisis de la ineficiencia de holgura. En el modelo 1, las DMU 2, 7, 8, 10, 11 y 12 tienen valores de holgura igual a cero porque sus eficiencias son iguales a 1 . Sucede lo mismo en el modelo 2 pero solo con las DMU 6 y 11 (Tabla 3).

Ahora bien, la más ineficiente en ambos modelos es la Finca Santa Teresita DMU 1, ésta presenta los valores de holgura más altos $32.08 \%$ y $80.26 \%$ respectivamente. Esto significa que tiene niveles de utilización de recursos por encima de los considerados como eficientes. En otras palabras, las variables de holgura en las entradas significan que las entradas deben ser reducidas en este valor de holgura para obtener la misma cantidad de salidas; y solo así podría llegar a un nivel de eficiencia igual a 1 (Tabla 3).

\section{Nivel objetivo de entradas y} fortalecimiento de las ineficiencias. Una vez determinada la eficiencia técnica relativa de cada finca, se consideran las ineficiencias de holgura con el fin de calcular sus niveles de entradas óptimos para lograr el nivel de eficiencia 1. Este cálculo se realiza tomando el valor inicial de las entradas y restarle el porcentaje del valor de holgura para obtener niveles óptimos de entradas para las DMU ineficientes.
Tabla 3. Valores de las holguras para los modelos 1 y 2.

\begin{tabular}{cccccc}
\hline & & \multicolumn{2}{c}{ Modelo 1 } & \multicolumn{2}{c}{ Modelo 2 } \\
\hline DMU & FINCAS & $\begin{array}{c}\text { V. } \\
\text { HOLGURA }\end{array}$ & \%EFIC & $\begin{array}{c}\text { V. } \\
\text { HOLGURA }\end{array}$ & $\%$ EFIC \\
\hline 1 & Santa Teresita & $32.08 \%$ & $67.92 \%$ & $80.26 \%$ & $19.74 \%$ \\
2 & El Recuerdo & $0 \%$ & $100 \%$ & $63.26 \%$ & $36.74 \%$ \\
3 & Amazonas & $28.22 \%$ & $71.78 \%$ & $51.01 \%$ & $48.99 \%$ \\
4 & El Rubí & $22.52 \%$ & $77.48 \%$ & $37.09 \%$ & $62.91 \%$ \\
5 & Casa Teja & $25.2 \%$ & $74.8 \%$ & $63.66 \%$ & $36.34 \%$ \\
6 & Los Pinos & $4.37 \%$ & $95.63 \%$ & $0 \%$ & $100 \%$ \\
7 & Los Arrayanes & $0 \%$ & $100 \%$ & $47.06 \%$ & $52.94 \%$ \\
8 & El Porvenir & $0 \%$ & $100 \%$ & $21.46 \%$ & $78.54 \%$ \\
9 & El Desvare & $55.88 \%$ & $70.72 \%$ & $79.81 \%$ & $20.19 \%$ \\
10 & Buena Vista & $0 \%$ & $100 \%$ & $52.83 \%$ & $47.17 \%$ \\
11 & El Molino & $0 \%$ & $100 \%$ & $0 \%$ & $100 \%$ \\
12 & El Dorado & $0 \%$ & $100 \%$ & $32.91 \%$ & $67.09 \%$ \\
\hline
\end{tabular}

Por ejemplo, en el modelo 1 la DMU 1 es la más ineficiente (Finca Santa Teresita), presentó un valor de holgura del $32.08 \%$ (Tabla 3); esta finca, tiene valores iniciales de entrada para nutrición, mantenimiento y ordeño de $\$ 93.466 ; \$ 12.558$ y $\$ 53.600$ correspondientemente (Tabla 4). En efecto, el reporte de nivel objetivo de entradas arroja los valores: \$63.477, \$8.529 y $\$ 36.402$ para nutrición, mantenimiento y ordeño respectivamente (Tabla 5); que es precisamente, tomar los valores iniciales y restarles el porcentaje arrojado por el valor de holgura de esta finca.

De esta manera, la Finca Santa Teresita cumpliendo con los niveles óptimos arrojados

Tabla 4. Valores iniciales en pesos modelos 1 y 2 en términos de miles de pesos (\$)

\begin{tabular}{ccccccc}
\hline & & \multicolumn{3}{c}{ Entradas Modelo 1 } & \multicolumn{2}{c}{ Entradas Modelo 2 } \\
DMU & FINCAS & Nutrición & Mantenim & Ordeño & Nutrición & Mantenim \\
\hline 1 & Santa Teresita & 93.466 & 12.558 & 53.600 & 93.466 & 12.558 \\
2 & El Recuerdo & 122.455 & 7.766 & 46.366 & 122.455 & 7.766 \\
3 & Amazonas & 66.010 & 11.739 & 54.041 & 66.010 & 11.739 \\
4 & El Rubí & 69.437 & 13.069 & 41.138 & 69.437 & 13.069 \\
5 & Casa Teja & 52.479 & 10.432 & 40.770 & 52.479 & 10.432 \\
6 & Los Pinos & 65.875 & 9.020 & 42.611 & 65.875 & 9.020 \\
7 & Los Arrayanes & 49.858 & 11.433 & 57.691 & 49.858 & 11.433 \\
8 & El Porvenir & 55.833 & 8.083 & 56.916 & 55.833 & 8.083 \\
9 & El Desvare & 124.250 & 15.250 & 38.687 & 124.250 & 15.250 \\
10 & Buena Vista & 51.138 & 10.861 & 40.500 & 51.138 & 10.861 \\
11 & El Molino & 64.333 & 13.760 & 33.104 & 64.333 & 13.760 \\
12 & El Dorado & 49.986 & 16.583 & 47.569 & 49.986 & 16.583 \\
\hline
\end{tabular}


Tabla 5. Niveles óptimos de entradas modelos 1 y 2

\begin{tabular}{ccccccc}
\hline & & \multicolumn{2}{c}{ Niveles Óptimos de Entradas Modelo $\mathbf{1}$ (\$) } & \multicolumn{2}{c}{ Niveles Óptimos de Entradas Modelo 2 (\$) } \\
DMU & FINCAS & Nutrición & Mantenim. & Ordeño & Nutrición & Mantenim. \\
\hline 1 & Santa Teresita & 63.477 & 8.529 & 36.402 & 12.129 & 2.479 \\
2 & El Recuerdo & 122.455 & 7.766 & 46.366 & 13.340 & 2.853 \\
3 & Amazonas & 47.382 & 8.426 & 38.791 & 32.339 & 4.428 \\
4 & El Rubí & 53.799 & 10.126 & 31.874 & 43.686 & 7.791 \\
5 & Casa Teja & 39.254 & 7.803 & 30.496 & 19.070 & 3.288 \\
6 & Los Pinos & 62.999 & 8.626 & 40.751 & 65.875 & 9.020 \\
7 & Los Arrayanes & 49.858 & 11.433 & 57.691 & 26.394 & 3.736 \\
8 & El Porvenir & 55.833 & 8.083 & 56.916 & 31.233 & 6.348 \\
9 & El Desvare & 54.820 & 10.785 & 27.359 & 19.638 & 3.079 \\
10 & Buena Vista & 51.138 & 10.861 & 40.500 & 24.119 & 3.900 \\
11 & El Molino & 64.333 & 13.760 & 33.104 & 64.333 & 13.760 \\
12 & El Dorado & 49.986 & 16.583 & 47.569 & 33.536 & 4.592 \\
\hline
\end{tabular}

por el reporte de niveles de entradas óptimos (Tabla 4), llegaría a tener una eficiencia igual a 1. De igual manera, se debe hacer el análisis anterior para las demás DMU ineficientes de los modelos 1 y 2 ; para que puedan llegar a su máximo nivel de eficiencia (Eficiencia $=1$ ).

Casos de éxito. Son aquellas fincas que fueron totalmente eficientes (Eficiencia=1), en los dos modelos. Es decir, no solo fueron eficientes en la producción de leche, sino que también lo fueron en la producción de carne y crías.

Por lo tanto, de acuerdo a los resultados arrojados por DEA solo hubo una coincidencia en los dos modelos, la DMU 11 que corresponde a la Finca El Molino. Entonces, la DMU 11 sería el único caso de éxito de la evaluación de la eficiencia técnica relativa de las fincas asociadas a la Cooperativa Coounión (Tabla 1). Esta DMU cuenta con las mejores prácticas en producción de leche, carne y crías. Por esta razón, se utilizará como referencia para medir la eficiencia técnica relativa de las demás DMU; y al mismo tiempo, se aplicarán estas buenas prácticas a las DMU ineficientes para mejorar sus niveles desempeños.

\section{DISCUSIÓN}

La medición de la eficiencia de las fincas asociadas a COOUNIÓN ha permitido identificar los niveles promedios de eficiencia para cada modelo; y con esto, identificar las buenas prácticas que permitan mejorar el nivel de eficiencia actual de las fincas que aún no son totalmente eficientes. Esto es importante para las fincas de la sabana de Bogotá, en donde los cambios climáticos han quemado los pastos, haciendo que la nutrición para el ganado sea cada vez más costosa y como consecuencia el ganadero deba ser más eficiente haciendo combinaciones óptimas de alimentos para sus animales (Tabla 1 ). El valor promedio de la eficiencia para el modelo 1 es del $88 \%$, significa que las fincas podrían producir la misma cantidad de producto con el $88 \%$ de las entradas que actualmente utiliza; es decir, están sacrificando un margen de ahorro del $12 \%$. En el modelo 2, las cifras son menos alentadoras porque la producción actual la podrían hacer utilizando solo el $56 \%$ de las entradas actuales y estarían ahorrando un margen del 44\% (Tabla 1 ).

De hecho, lo anterior es la consecuencia de la especialización en la producción de leche del $80 \%$ de las fincas, actividad a la que dirigen todos sus recursos y esfuerzos. En cambio, la producción de carne y crías lo trabajan alternamente para generar ingresos adicionales no muy representativos. Según los propietarios la variable que más pesa es la nutrición con el $70 \%$ de los costos, que está orientada a aumentar la producción de leche. Razón 
por la cual, son más eficientes en su producción, que en la producción de carne y crías.

Es definitivo que la mejor práctica es el mejoramiento genético. Esto se observa en la Finca El Molino (DMU 11) con eficiencia total en ambos modelos, porque hace mejoramiento genético orientado a razas lecheras europeas como Holstein, Jersey y Pardo Suizo. Es así, que cuenta con tan solo 4 vacas y tiene un promedio de 455 litros de leche por vaca al mes. Por ende, sus crías son demandadas para destinarlas a la producción de leche en Guasca-Cundinamarca (Tabla 2).

Asimismo, la DMU 6 sobresalió por ser la mayor generadora de ingresos por venta de ganado en pie (carne) (Tabla 2), también debe su éxito al mejoramiento genético orientado al ganado de doble propósito (leche y carne) ganado Simmental, que años atrás ha venido implementando en su hato ganadero. Por eso, el mejoramiento genético en ganado doble propósito es una buena práctica que posiciona esta finca entre las mejores del estudio de caso.

Por consiguiente, se observa en el modelo 1 y 2 que los niveles óptimos de entradas arrojados para cada DMU por DEA, se deben lograr para que aquellas fincas con ineficiencias a escala puedan alcanzar un nivel de eficiencia 1 (Tabla 5). Estos niveles óptimos de entradas para las DMU con eficiencia 1, son los mismos valores de entradas iniciales; esto se debe, a que sus valores de holgura son iguales a cero (Tabla 4). Por este motivo, las reducciones en las entradas se dan para las DMU ineficientes, las cuales deben hacer disminuciones en los recursos para llegar a los niveles óptimos de insumos conservando el mismo nivel de producción, y así lograr el nivel de eficiencia 1.
Por ende, se recomienda DEA para futuros estudios de evaluación de eficiencia en fincas ganaderas. Porque permite medir la eficiencia técnica relativa concentrándose bien sea en los insumos o en los productos según convenga para el caso. Generalmente, estos estudios se enfocan en los insumos porque son más controlables. De ahí que, las variables de salida están mediadas por factores que no son controlables por los ganaderos; tales como: el clima, las políticas del Gobierno, las enfermedades en los bovinos y hasta el mismo conflicto armado que ha sido el principal flagelo de la industria ganadera en Colombia.

Finalmente, como limitaciones del DEA se tiene en primera instancia que la comparación de los resultados de dos estudios aplicados a grupos de muestras diferentes no es significativo; puesto que se desconocen las diferencias existentes entre las prácticas empleadas por las DMU de cada una de las muestras. Y en segunda instancia, se tiene también que los resultados son altamente sensibles a la presencia de errores de medición en las entradas y salidas (21).

En conclusión, la evaluación de la eficiencia técnica relativa realizada a las doce fincas, se hizo orientado a las entradas lo que permitió demostrar que las fincas podrían mantener sus niveles de producción o salidas haciendo una reducción en sus entradas o insumos.

\section{Agradecimientos}

A la Cooperativa Coounión y en especial a su gerente Nilson Morales quien dispuso de todo su tiempo y su conocimiento, para brindar la información necesaria para realizar esta investigación. 


\section{REFERENCIAS}

1. Durán F. Volvamos al campo. Manual del ganadero actual Tomo I. Bogotá: Grupo Latino; 2004.

2. Bonnett D. Entre el interés personal y el establecimiento colonial: factores de confrontación y de conflicto en el Nuevo Reino de Granada entre 1538 y 1570. Hist Crit 2009; 39(1):52-67.

3. Federación Colombiana de Ganaderos. La ganadería bovina en Colombia. Bogotá: Fondo de Publicaciones FEDEGAN; 2004.

4. Naves M, Laloe D, Goudarzi K, Debus A. Relaciones genéticas entre el bovino criollo de Guadalupe y otras razas por marcadores bioquímicos. Arch Zootec 2005; 54:385-394.

5. Marulanda O. Labranza de paz. Producción sostenible en el campo para la reconstrucción de la comunidad rural. Colombia siglo XXI: realidad y cambio. Bogotá: Grupo Editorial Norma; 2002.

6. Gore A. Una verdad incómoda. [Video] EUA: Paramount Classics; 2006.

7. Gutiérrez S. Cambio climático. Revista Dinero 2006; 246:56.

8. Camargo J. El libro de la finca. Un práctico manual para las labores del campo. Bogotá: Casa Editorial El Tiempo; 2007.

9. Rodríguez G, Arcelus F. Modelo no paramétrico aplicado a la medición de eficiencia de la extensión universitaria, innovación aplicable a Latinoamérica. Rev Humanid Cienc Soc 2009: 15:43-68.

10. Rodríguez G. Medición de la eficiencia relativa en dos subsectores de la economía colombiana desde 1993 a 2002 utilizando Data Envelopment Analysis (DEA). Innovar 2003; 22:121-144.
11. Ruiz A. Aplicación del método de optimización DEA en las evaluación de la eficiencia técnica de las seccionales de la fiscalía. Documentos CEDE 2004; 1-26. URL Disponible En: http://economia. uniandes.edu.co/investigaciones_y_ publicaciones/CEDE/Publicaciones/ documentos_cede/2004/aplicacion_ del_metodo_de_optimizacion_dea_ en_la_evaluacion_de_la_eficiencia_ tecnica_de_las_seccionales_de_la_ fiscalia

12. Cook W, Seiford L. Data Envelopment Analysis (DEA) - Thirty years on. Eur J Oper Res 2008, 192:1-17.

13. Lanski A, Reinhard S. Investigating technical efficiency and potential technological change in Dutch pig farming. Agric Syst 2004; 79(3):353-382.

14. Hernangómez J, Martín N, Pérez V. Implicaciones de la organización interna sobre la eficiencia. La aplicación de la teoría de la agencia y la metodología DEA a las ONGD españolas. Revista CEDE 2009; 40:17-46.

15. Gaspar P, Mesías F, Escribano M, Pulido $F$. Assessing the technical efficiency of extensive livestock farming systems in Extremadura, Spain. Livest Sci 2009; 121:7-14.

16. Gamarra J. Microeconomía de la ganadería en Colombia. Eficiencia técnica relativa de la ganadería doble propósito en la Costa Caribe. Colección de Economía Regional. Bogotá: Banco de la República; 2005.

17. Coll $V$, Blasco O. Evaluación de la eficiencia mediante el Análisis Envolvente de Datos. Introducción a los Modelos Básicos. Madrid: Universidad de Valencia; 2006. 
18. Casierra F, Rodríguez J, Cárdenas J. La relación hoja: fruto afecta la producción, el crecimiento y la calidad del fruto en duraznero (Pronus pérsica L. Batsch, cv. 'Rubidoux'). Rev Fac Nal Agr Medellin 2007; 60:57-69.

19. Martínez H. La cadena de lácteos en Colombia. Una mirada global de su estructura y dinámica 1991-2005. Observatorio Agrocadenas Colombia [en línea]. 2005. [fecha de acceso 17 de junio de 2010]; URL Disponible En: http://www.redlactea.org/documentos/ Inf\%20coy\%201991\%202005.pdf
20. Welcome to Joe Zhu's Homepage [en línea]. USA: Dea Frontier; 2007. [fecha de acceso 7 de mayo de 2010]. URL disponible en: http://www.deafrontier. net/joezhu/index.html

21. Rodríguez Guzmán E. Aplicación de la metodología DEA para el establecimiento de índices de eficiencia para empresas de distribución de energía. [Tesis]. Bogotá: Facultad de Ingeniería Electríca, Universidad Nacional de Colombia; 2007. 